With a laser rod having high-reflectivity mirrors on both ends, threshold was reached at a rod temperature of $3.5{ }^{\circ} \mathrm{C}$ at $250 \mathrm{~mA} /$ diode.

The dotted portion of the curve in Fig. 3 is an extrapolation, based on Fig. 2, to a rod temperature of $20^{\circ} \mathrm{C}$. In these experiments we were limited to an array of 19 diodes by the dimensions of the case in which the diodes were mounted rather than the size of the emitting elements themselves. With a more compact diode package the number of diodes could be increased by a factor of $3-4$. Using Fig. 3 to extrapolate, a rod at $20^{\circ} \mathrm{C}$ pumped by 60 diodes would be 2.2 times above threshold and when pumped by 80 diodes would be 2.9 times above threshold.

The optical pump power would be 3 to 4 times higher than in the present experiments and the laser rod would undoubtedly have to be more efficiently heat sunk. This could be readily accomplished by coating half the outer surface of the rod with gold and thermally bonding it into a channel in the heat sink. This would represent a considerable improvement in the conductivity between the rod and heat sink, and no heating problem is anticipated at these higher power levels. Therefore, we conclude that a $\mathrm{cW}$ diode pumped YAG : Nd laser operating at room temperature $\left(20^{\circ} \mathrm{C}\right)$ with a power output $\approx 50 \mathrm{~mW}$ can be built with presently available $4 \%$ efficient $\mathrm{GaAs}_{1-x} \mathrm{P}_{x}$ diodes.

I am very grateful to J. E. Geusic for advice and encouragement throughout this work and to D. R. Mackenzie for his excellent mechanical designs. I also thank P.H. Buhite for writing a computer program.

\footnotetext{
${ }^{1}$ R. J. Keyes and T. M. Quist, Appl. Phys. Letters $\underline{4}$, 50 (1964).

${ }^{2}$ S. A. Ochs and J.I. Pankove, Proc. IEEE $\underline{52}, 713$ (1964).

${ }^{3}$ M. Ross, Proc. IEEE 56, 196 (1968).

${ }^{4}$ R. B. Allen and S. J. Scalise, Appl. Phys. Letters 14, 188 (1969).

${ }^{5}$ S. J. Scalise and R.B. Allen, Air Force Avionics Laboratory Technical Report AFAL-TR-70-49, 1970 (unpublished).

${ }^{6}$ The diodes were designated by the code OSX1507 (without reflector) together with the wavelength desired.

${ }^{7} R$ and $Y$ refer to the ${ }^{4} F_{3 / 2}$ and ${ }^{4} I_{11 / 2}$ manifolds, respectively, and the Stark levels within a manifold are numbered consecutively from 1 starting with the lowest energy level.

${ }^{8}$ T. Kushida, Phys. Rev. $\underline{185}, 500$ (1969).
}

\title{
PARAMETER DEPENDENCE OF ACOUSTOELECTRIC AMPLIFICATION IN InSb ${ }^{\dagger}$
}

\author{
W. J. Fleming and J. E. Rowe \\ Electron Physics Laboratory, Department of Electrical Engineering, \\ University of Michigan, Ann Arbor, Michigan 48104
}

(Received 28 September 1970)

\begin{abstract}
On the basis of a hydrodynamical theory of the acoustoelectric interaction (Fleming-Rowe) reported earlier which included electron inertial terms it is found that for sufficiently large electron drift velocities sharp high-gain peaks occur. Furthermore the peak values of gain achieved greatly exceed the maximum gain of the corresponding theory of Steele. Excellent agreement with recently reported experimental measurements of mic rowave acoustic gain in InSb is obtained. It is also noted that for large applied fields, empirical field factors are required to give agreement with experiment.
\end{abstract}

Recently it has been concluded by several investigator $\mathrm{s}^{1-3}$ that detailed microscopic theories are required to adequately account for microwave acoustoelectric effects in InSb. It has been shown by the author $\mathrm{s}^{4}$ that, when electron intertial terms are retained, a hydrodynamical theory of the acoustoelectric interaction gives valuable insight into the interaction process. When this theory is modified to include empirical field factors excellent agreement with experimental measurements of microwave acoustic gain ${ }^{1,5}$ is obtained.

The derivation and notation of the hydrodynamical theory developed by the authors followed that of other related work. ${ }^{6-8}$ Retaining electron inertial terms, we find that the acoustoelectric growth rate, occurring in the presence of a transverse magnetic field $B_{1}$, is ${ }^{4}$

$$
\alpha=-\kappa^{2} \frac{\omega_{R}^{\prime}}{2 v_{s}} \frac{\gamma}{\left(\gamma^{2}+W^{\prime 2}\right)},
$$

where $\kappa^{2}$ is the electromechanical coupling constant, $v_{s}$ is the sound wave velocity, $\gamma=1-u_{o} / v_{s}$, where $u_{o}$ is the electron drift velocity, $W^{\prime}=\omega_{R}^{\prime} / \omega$ $+\omega / \omega_{D}^{\prime}, \omega_{R}^{\prime}=\omega_{R} / \nu_{\mathrm{R}}^{\prime}$ is the effective dielectric relaxation frequency, $\omega_{D}^{\prime}=\omega_{D} \nu_{\mathrm{Re}}^{\prime} /\left(1-\gamma \nu_{\mathrm{Im}}^{\prime} \omega_{D} / \omega\right)$ is the effective diffusion frequency, $\omega_{R}=\sigma_{0} / \epsilon, \omega_{D}=\nu v_{s}^{2} / \nu_{T}^{2}$, $\nu$ is the electron relaxation frequency, $v_{T}$ is the electron thermal velocity, $\nu_{\mathrm{Re}}^{\prime}=1+b^{62}, \nu_{\mathrm{Im}}^{\prime}=\omega_{\nu}(1-$ $\left.b^{22}\right), b^{\prime 2}=b^{2} /\left(1+\omega_{v}^{2}\right)$, where $b$ is the dimensionless $\mu B_{\perp}$ product, and $\omega_{\nu}=\omega \gamma / \nu$ is the parameter which 
includes the effect of electron inertial terms. When interial terms are neglected, $\omega_{\nu}-0$, (1) reduces to the corresponding result of Steele ${ }^{6}$ When the magnetic field is zero, $b \rightarrow 0$, (1) reduces to the result of Kino and Route ${ }^{7}$ and when both inertial terms and the magnetic field effects are neglected, $\omega_{\nu} \rightarrow 0$ and $b \rightarrow 0$, (1) reduces to the result of White. ${ }^{8}$

Abe and Mikoshiba ${ }^{9}$ have previously considered the case $\omega_{\nu} \neq 0$ and $b \neq 0$; however, their results are in error. When $b=0$, their hydrodynamical theory does not reduce to the results of Kino and Route. ${ }^{7}$ The discrepancy arises from the specialized magnetohydrodynamic expression [Eq. (1) of Ref. 9] which they use for the equation of momentum conservation. This equation is given in terms of current rather than electron velocity and is taken from Eq. (2-12) of Spitzer. ${ }^{10}$ Spitzer points out however that Eq. $(2-12)$ is subject to the approximation that "... deviations from electrical neutrality... are ignored in the dynamical equations for $\overrightarrow{\mathrm{J}}$..."; it appears that Abe and Mikoshiba have incorrectly introduced the space-charge term $Q_{e} n_{1} \overrightarrow{\mathbf{u}}_{0}$ into this equation whereupon the erroneous term $\omega_{\nu} u_{0} / v_{s}$ arises. ${ }^{11}$

Acoustic gain is calculated from (1) using the relation, gain $=10 \log _{10}[\exp (2 \alpha)]=8.68 \alpha(\mathrm{dB} / \mathrm{cm})$. The results of this calculation are shown in Fig. 1, where it is seen that no significant error is present in the theory of Abe and Mikoshiba ${ }^{9}$ for electron drift velocities less than approximately $3 \times$ $10^{6} \mathrm{~cm} / \mathrm{sec}$. However at large drift velocities, the theory of Abe and Mikoshiba noticeably diverges from the theory of the present paper as a result of their error. The locations of the sharply peaked maximum gain values which exist for $u_{0}>v_{T}$ can be determined directly from (1). They occur when the condition $W^{\prime}=0$ is satisfied and this condition is given, in good approximation, by

$$
\gamma^{2}=\left(v_{T}^{2} / v_{s}^{2}\right)\left[1+\left(\omega_{0} / \omega\right)^{2}\left(1+b_{R}^{2}\right)\right],
$$

where $\omega_{0}=\left(\omega_{R} \omega_{D}\right)^{1 / 2}=2 \pi f_{0}$ and $b_{R}^{2}=b^{2} \nu / \omega_{R}$. Physi-



FIG. 1. Acoustic gain in $n$-InSb for $\kappa^{2}=1 \times 10^{-3}$, $\mu_{0}=6 \times 10^{5} \mathrm{~cm}^{2} / \mathrm{V} \mathrm{sec}$, and $N_{0}=1 \times 10^{14} \mathrm{~cm}^{-3}$.

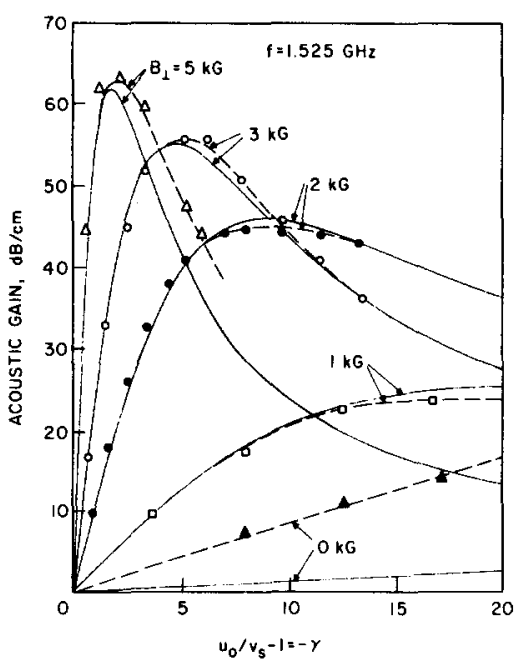

FIG. 2. Comparison between hydrodynamical theory and the Route-Kino experiment for $\kappa^{2}=1.3 \times 10^{-3}$, $\mu_{0}=5.1 \times 10^{5} \mathrm{~cm}^{2} / \mathrm{V} \mathrm{sec}$, and $N_{0}=1.4 \times 10^{14} \mathrm{~cm}^{-3}$ 'solid curves denote theoretical results).

cally, it can be shown that (2) corresponds to cancellation of the diffusion term by the electron inertial term in the equation of momentum conservation. From (1) and (2) maximum gain is given, in good approximation, by

$(\text { gain })_{\max }=8.68 \kappa^{2} \frac{\omega_{R}}{2 v_{T}} \frac{\left[1+\left(\omega_{0} / \omega\right)^{2}\left(1 L h_{R}^{2}\right)\right]^{1 / 2}}{\left[1+\left(\omega_{0} / \omega\right)^{2}\left(1+2 b_{R}^{2}\right)\right]}(\mathrm{dB} / \mathrm{cm})$

At the frequency $f=f_{0}=1.45 \mathrm{GHz}$, substitution of the parameters of Fig. 1 into (2) and (3) yields (gain) $_{\max }=623 \mathrm{~dB} / \mathrm{cm}$ which occurs at $u_{0}=4.26 \times$ $10^{7} \mathrm{~cm} / \mathrm{sec}$ for $b=0$. This value of maximum gain is 14.7 times greater than the corresponding maximum gain given by Steele's theory, ${ }^{6}$ however, it can only occur for $u_{0}>v_{T}$ and $b \simeq 0$. Under these conditions hot electron effects and microscopic resonance effects must be considered. Notwithstanding, extensive numerical calculations ${ }^{4}$ have shown that at more reasonable drift velocities $\left(u_{0} \leqslant 0.8 v_{T}\right)$, the essential features of the corresponding microscopic theories ${ }^{1-3}$ are retained in the present hydrodynamical theory. In particular, analysis of the present theory indicates that the Mode I acoustic domain formation (which occurs for $u_{0} \gtrsim 0.4 v_{T}$ and $b \simeq 0$ ) does not critically depend on microscopic resonance effects; but rather, it arises from the same physical mechanism which gives rise to the sharp gain peaks of Fig. 1, namely, a drift-enhanced quenching of electron diffusion effects.

In Fig. 2 the theoretical acoustic characteristics are calculated from (1) and are compared to the experimental results of Route and Kino. ${ }^{1}$ The 


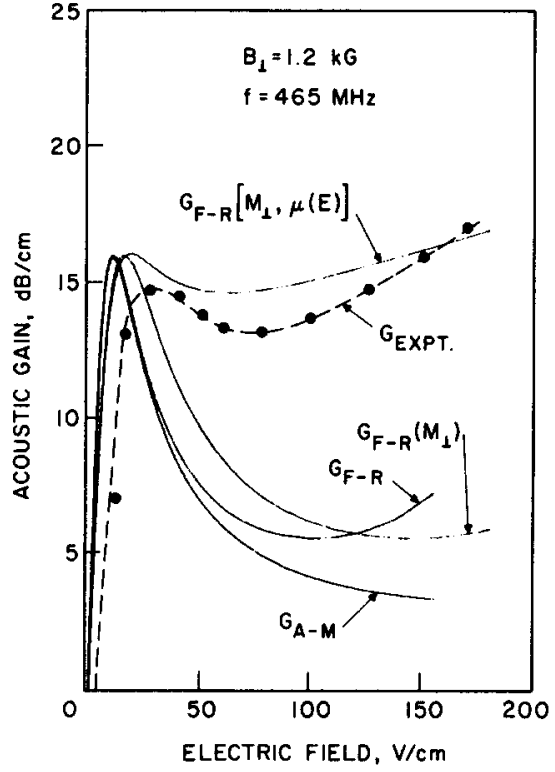

(a)

agreement is excellent except for the zero magnetic field curve. Although the microscopic theory ${ }^{1}$ gives better agreement for the zero magnetic field curve, the hydrodynamical theory of the present paper gives as good, if not better, agreement for the remaining $\left(B_{1} \neq 0\right)$ acoustic gain curves.

Under other conditions, field-dependent effects such as electron mobility saturation and geometrical magnetoresistance are important. An empirical relation for the mobility saturation is obtained by a least-squares fit to the $I-V$ characteristic data. The $I-V$ data for three specimens of high-purity $n$-type InSb was measured at $77^{\circ} \mathrm{K}$ and a least-squares fit of the resistivity function,

$$
\rho=\rho_{0}\left(1+\alpha_{2} J_{0}^{2}+\alpha_{4} J_{0}^{4}\right)=\rho_{0} F\left(J_{0}\right),
$$

is obtained using 120 data points measured at $B_{\perp}=0$ for $\left|J_{0}\right| \leqslant 400 \mathrm{~A} / \mathrm{cm}^{2}$ and $\left|E_{0}\right| \leqslant 130 \mathrm{~V} / \mathrm{cm}$. The values $\alpha_{2}=6.87 \times 10^{-6} \mathrm{~cm}^{4} / \mathrm{A}^{2}$ and $\alpha_{4}=3.15 \times 10^{-13}$ $\mathrm{cm}^{8} / \mathrm{A}^{4}$ are obtained. ${ }^{12}$ The parameter $\alpha_{2}$ can be compared to the parameter $\beta$ in the conductivity relation $\sigma=\sigma_{0}\left(1+\beta E_{0}^{2}\right)$. The parameter $\beta$ is equal to $-\alpha_{2} \bar{\sigma}_{0}^{2}$, where $\bar{\sigma}_{0}=6.84(\Omega-\mathrm{cm})^{-1}$ is the average low-field conductivity of the measurement. A value of $\beta=-3.22 \times 10^{-4} \mathrm{~cm}^{2} / \mathrm{V}^{2}$ is obtained, which is in agreement with the range of $-3.5<\beta<-1 . j \times 10^{-4}$ $\mathrm{cm}^{2} / \mathrm{V}^{2}$ determined by George and Bekefi. ${ }^{13}$ The empirical relation for geometrical magnetoresistance is obtained by matching boundary conditions with the results of a theoretical analysis. ${ }^{14}$ The constants $\delta_{1}, \delta_{2}$, and $\delta_{3}$ for the assumed magnetoresistance function (valid for $L \gg w$ and $b \geqq 0$ )

$$
g(l)=\delta_{1} l+\delta_{2} l^{2}+\delta_{3} l^{3}=(L / w b)\left[M_{1}(b)-1\right],
$$

are determined by matching the conditions $g(1)=1$,

$$
\lim _{b^{2} \ll 1} g(l)=14 b S_{3} / \pi^{3},
$$

and

$$
\lim _{b^{2} \gg 1} g(l)=1-4(\ln 2) / \pi b,
$$

where $l=2\left(\tan ^{-1} b\right) / \pi, S_{3}=1.2021$ is a numerical constant, $L$ and $w$ are the length and width of the specimen, and $M_{\perp}(b)=R_{0}(b) / R_{0}(0)$ is the magnetoresistance ratio. The values $\delta_{1}=0.8526, \delta_{2}=$ -0.0915 , and $\delta_{3}=0.2389$ are obtained.

The empirical field factors given by (4) and (5) are incorporated into (1) in the following manner. Specifying an electron drift velocity $u_{0}$, the applied current and applied electric field are determined by

$$
J_{0}=Q_{e} N_{0} u_{0} \text { and } E_{0}=\left(u_{0} / \mu_{0}\right) \cdot M_{\downarrow}(b) \cdot F\left(J_{0}\right)
$$

where $\mu_{0}$ is the low-field electron mobility. ${ }^{15}$ Assuming that the field-dependent electron mobility varies as $\mu=\mu_{0} / F\left(J_{0}\right)$, the relaxation parameters in (1) become $\omega_{R} \rightarrow \omega_{R} / F\left(J_{0}\right), \omega_{D} \rightarrow \omega_{D}^{\circ} F\left(J_{0}\right), \nu \rightarrow \nu$ - $F\left(J_{0}\right)$, and $b-b / F\left(J_{0}\right)$. The importance of these empirical relations is illustrated in Fig. 3(a). Experimental acoustic gain values are taken from the work of Hayakawa and Kikuchi. ${ }^{5}$ The curves labeled $G_{\mathrm{A}-\mathrm{M}}$ and $G_{\mathrm{F}-\mathrm{R}}$ are, respectively, the theoretical results of the theory of Abe and Mikoshiba ${ }^{9}$ and the present theory of Fleming and Rowe, where (in both cases) no empirical effects are included and the electric field is taken as $E_{0}=u_{0} / \mu_{0}$. Only the effect of magnetoresistance has been included in the curve $G_{\mathrm{F}-\mathrm{R}}\left(M_{1}\right)$ wherein $E_{0}=\left(u_{0} / \mu_{0}\right) \circ M_{1}(b)$. Finally, all empirical factors are included in the curve $G_{\mathrm{F}-\mathrm{R}}\left[M_{\perp}, \mu(E)\right]$, wherein the effects of mo- 
bility saturation are now included in the gain calculation and the electric field is given by (6). It is apparent that the empirical field factors must be included in order to obtain good agreement between experiment and theory.

In Fig. 3(b) the complete set of theoretical acoustic gain characteristics, including all the empirical field factors and using (1), are compared to the experimental results. ${ }^{5}$ Over-all good agreement is obtained. Deviations from the experimental results are attributed to discrepancies between the assumed empirical field factors of (6) and the actual field factors of the experiment.

\footnotetext{
'Work supported by the Air Force Systems Command's Rome Air Development Center.

${ }^{1}$ R. K. Route and G. S. Kino, IBM J. Res. Develop. $\underline{13}$, 507 (1969).

${ }^{2}$ K. P. Weller and T. Van Duzer, J. Appl. Phys. 40 , 4278 (1969) .

${ }^{3}$ W. Harth and R. Jaenicke, Appl. Phys. Letters 14, 27 (1969).

${ }^{4}$ W. J. Fleming and J.E. Rowe, J. Appl. Phys. (to be published).

${ }^{5}$ H. Hayakawa and M. Kikuchi, Appl. Phys. Letters 17, $73(1970)$.
}

${ }^{6}$ M. C. Steele, R C A Rev. 28, 58 (1967).

${ }^{7}$ G.S. Kino and R. Route, Appl. Phys. Letters 11 , 312 (1967).

${ }^{8}$ D. L. White, J, Appl. Phys. $\underline{33}, 2547$ (1962).

${ }^{9} \mathrm{Y}$. Abe and N. Mikoshiba, Appl. Phys. Letters $\underline{13}$, 241 (1968).

${ }^{10}$ L. Spitzer, Jr., Physics of Fully Ionized Gases (Interscience, New York, 1956).

${ }^{11}$ It can be shown that the factor $\omega / \omega_{D}=q D / v_{s}$ of the present theory (contained in the definition of $\omega / \omega_{D}^{\prime}$ ) and the theory of Kino and Route (Ref. 7) is modified and becomes $\omega / \omega_{D}=q D / v_{s}+\omega_{v} u_{0} / v_{s}$ in the theory of Abe and Mikoshiba (Ref. 9). The additional term $\omega_{v} u_{0} / v_{s}$ appearing in the theory of Abe and Mikoshiba is erroneous and comes directly from the specialized magnetohydrodynamic expression [Eq. (1) of Ref. 9] which they use for the equation of momentum conservation.

${ }^{12}$ The contribution of the quartic term in (4) is negligible for all but the largest values of applied current. For example, the value of the quartic term just exceeds $1 \%$ of the value of the quadratic term for $u_{0}=v_{T}\left(J_{0}=480\right.$ $\left.\mathrm{A} / \mathrm{cm}^{2}\right)$.

${ }^{13}$ E. V. George and G. Bekefi, Appl. Phys. Letters $\underline{15}$, 33 (1969)

${ }^{1}$ H.J. Lippman and F. Kuhrt, Z. Naturforsch. 13a, 462 (1958).

${ }^{15}$ Taking $I_{0}=J_{0} w^{2}$ and $V_{0}=E_{0} L, I-V$ characteristics calculated from (6) are a good approximation to the actual measured $I-V$ characteristics.

\title{
TYPE CONVERSION AND $p-n$ JUNCTION FORMATION IN LITHIUM-ION-IMPLANTED ZnSe
}

\author{
Y.S. Park \\ Aerospace Research Laboratories, Wright-Patterson AFB, Ohio 45433 \\ and \\ C. H. Chung* \\ Department of Physics, University of Dayton, Dayton, Ohio 45409 \\ (Received 5 October 1970)
}

\begin{abstract}
High-conductivity $n$-type ZnSe implanted with Li ions gives evidence of type conversion when tested by thermal probe measurements. Photovoltaic effect, current rectification, and low-voltage electroluminescence were observed in diodes fabricated from implanted material.
\end{abstract}

We have observed $p$-type conduction in Li-ionimplanted $n$-type ZnSe single crystals and formed $p-n$ junctions. Type conversion and $p-n$ junction formation by ion implantation have been recently produced in other similar II-VI compounds, $n$-CdS, ${ }^{1-3} n$-CdTe, ${ }^{4}$ and $p-\mathrm{ZnTe},{ }^{5}$ but this is the first report of successful type conversion produced by ion implantation in high-conductivity $n-\mathrm{ZnSe}$.

ZnSe crystals, as grown, usually exhibit highresistivity $n$-type conductivity. High resistivity is thought to arise from stoichiometric deficiency; possibly due to $\mathrm{Zn}$ vacancies produced during crystal growth. Undoped crystals of $\mathrm{ZnSe}$ grown from the melt by Eagle-Picher Co. were used in this experiment. As received, these crystals showed high resistivity in the range $10^{8}-10^{10} \Omega-\mathrm{cm}$ at room temperature. By firing in molten $\mathrm{Zn}$ for $24 \mathrm{~h}$ at $900^{\circ} \mathrm{C}$, we obtained low-resistivity crystals in the range of $0.1-10 \Omega-\mathrm{cm}$. A crystal having a resistivity of $3 \Omega-\mathrm{cm}$ had, for example, a roomtemperature electron mobility of $480 \mathrm{~cm}^{2} / \mathrm{V} \mathrm{sec}$ and a carrier concentration of $4 \times 10^{15} \mathrm{~cm}^{-3}$. Typical sample dimensions used for implantation were $13 \times 7 \times 1 \mathrm{~mm}$. Wafers were cleaved and $\langle 110\rangle$ oriented. Following heat treatment in molten $\mathrm{Zn}$, the implantation surface was optically finished and chemically etched for several seconds in concentrated boiling $\mathrm{NaOH}$.

All implantations ${ }^{6}$ were carried out at room temperature. Li ions were supplied from the source material of beta-eucryptite ${ }^{7}$ pasted on the tungsten filament in the ion source. The mass analyzer was 